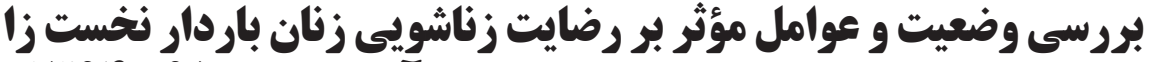

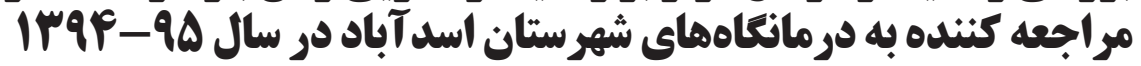

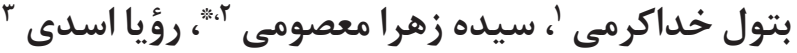

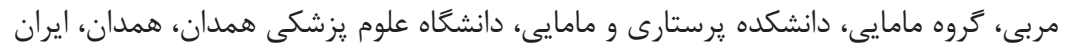

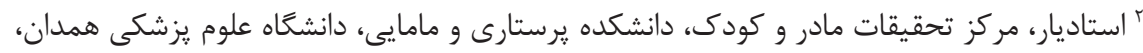

$$
\text { همدان، ايران }
$$

× دانشجو، كروه مامايى، دانشكده يُرستارى مامايى، دانشعاه علوم يزشكى همدان، همدان، ايران

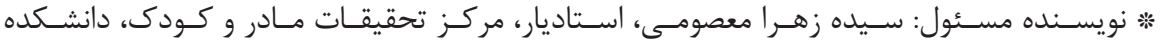

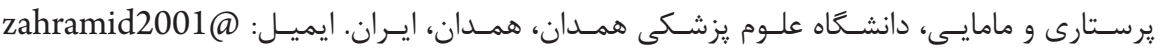

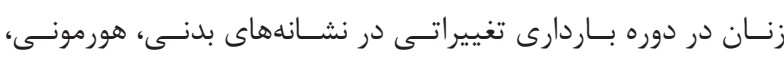

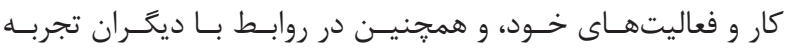

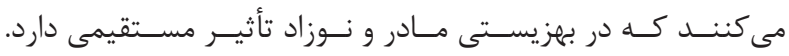

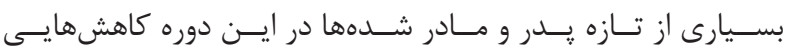

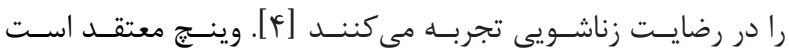

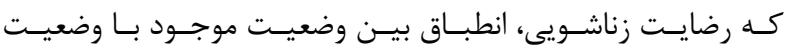

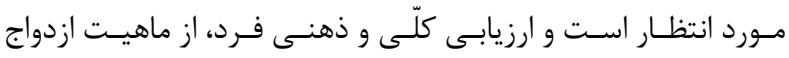

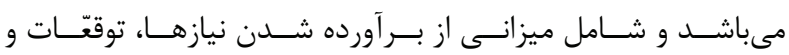

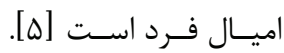

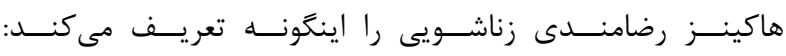

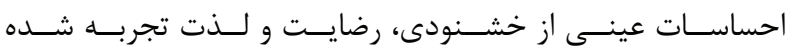

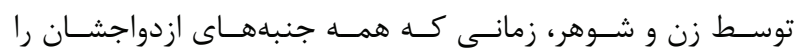

بــاردارى و زايمـــان بــهـه عنـــوان يـــ رويــداد طبيعــى در دوران

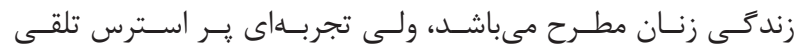

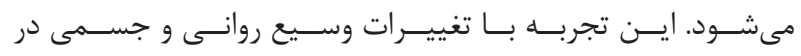

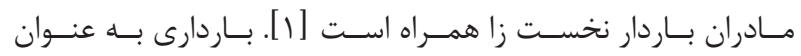

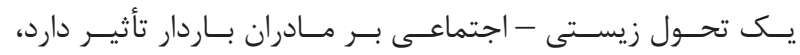

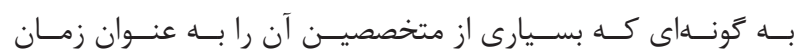

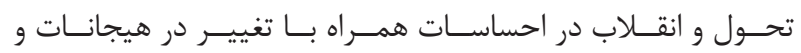

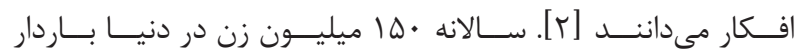

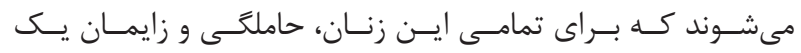

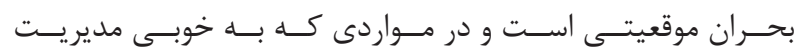

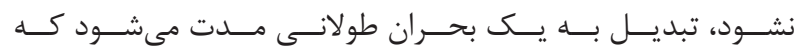

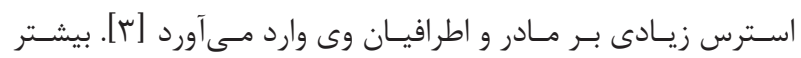




\section{روش كار}

نــوع مطالعـه توصيفى -تحليلـى اسـت كـهـ بــهـ بررسـى ميـزان رضايت

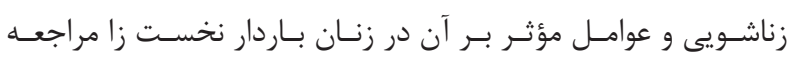

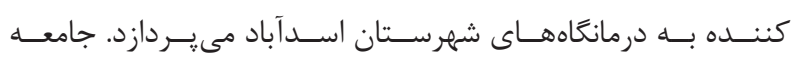

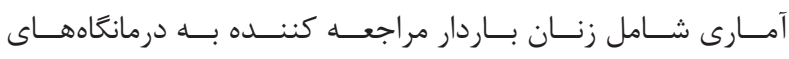

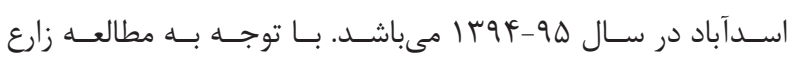

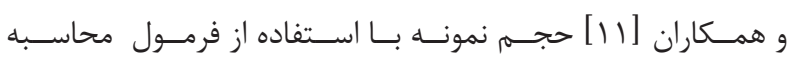

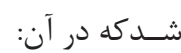

N تعداد كل جامعه آمارى t = IVD d تقريب برآورد يارامتر مورد مطالعه

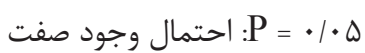

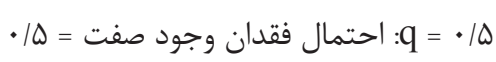

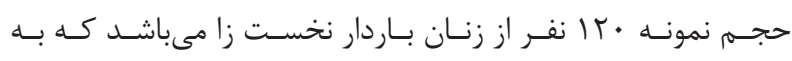

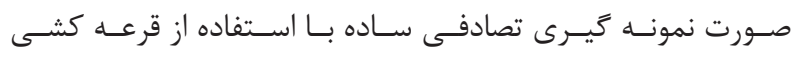

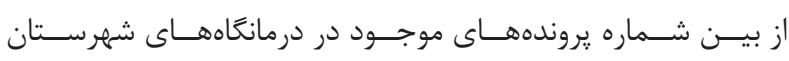

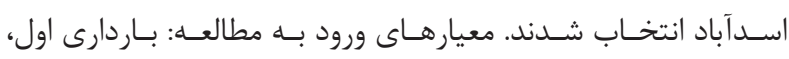

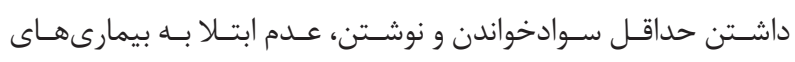

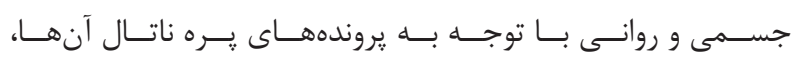

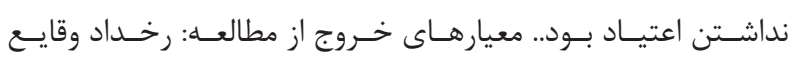

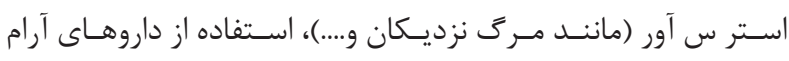

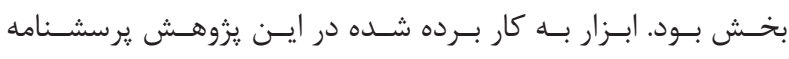

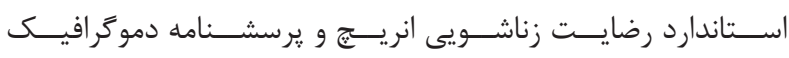

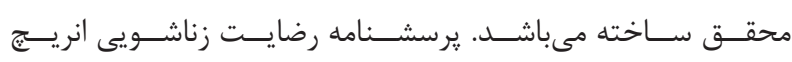

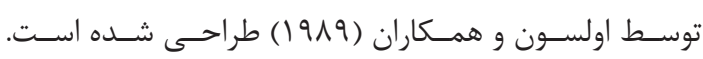

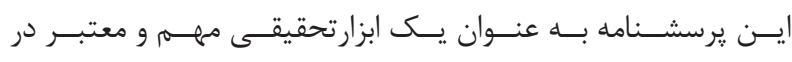

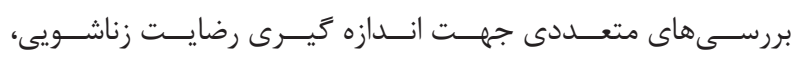

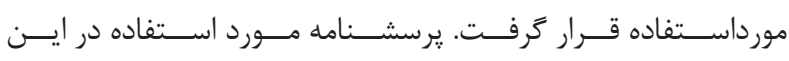

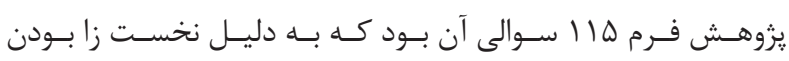

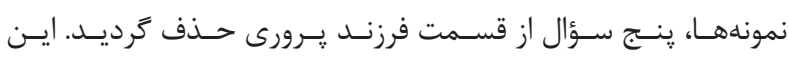

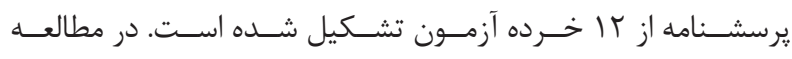

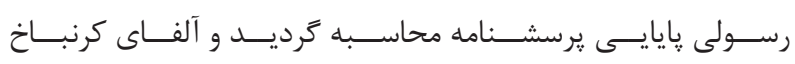

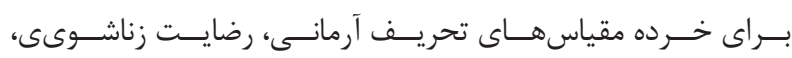

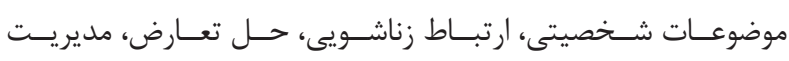

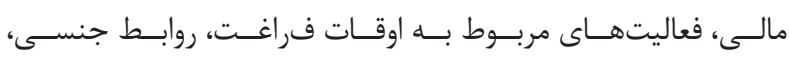

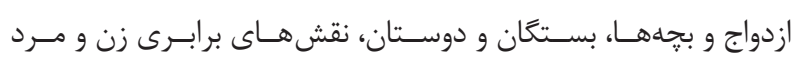

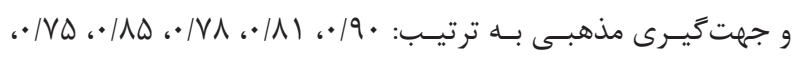
ف

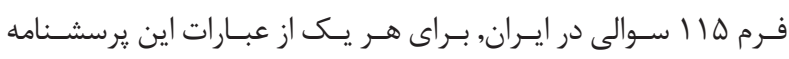

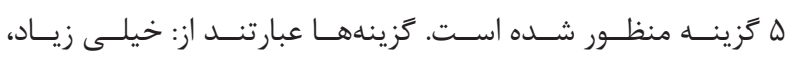

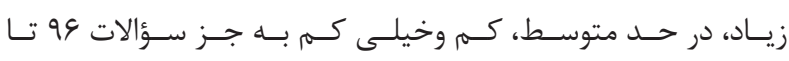

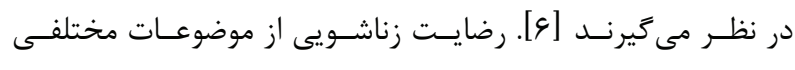

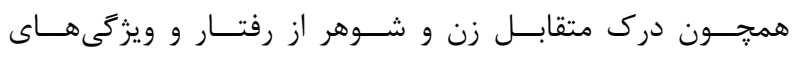

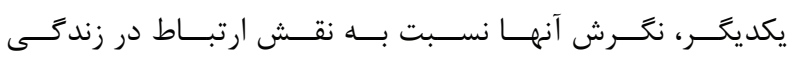

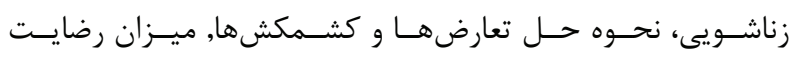

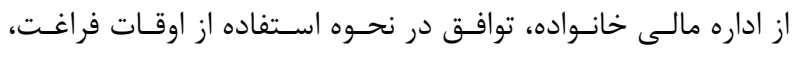

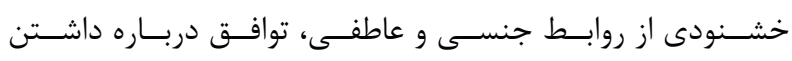

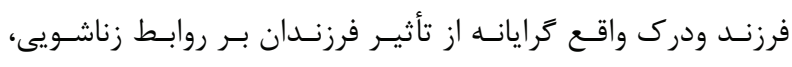

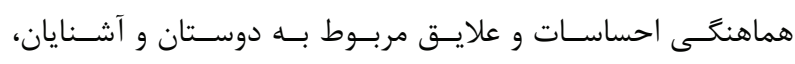

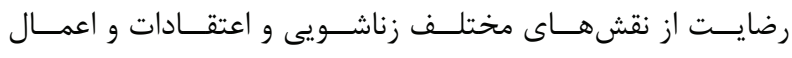

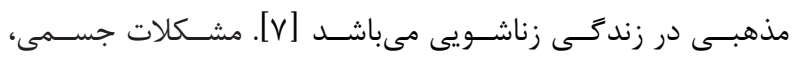

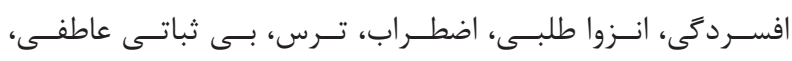

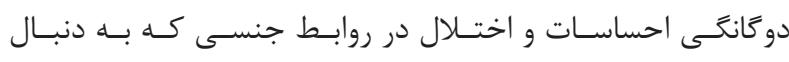

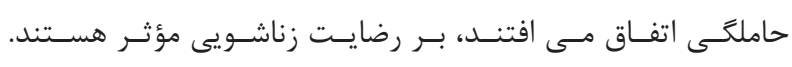

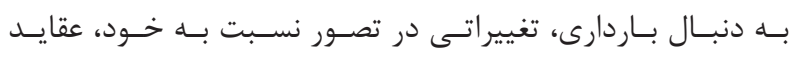

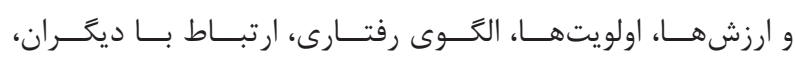

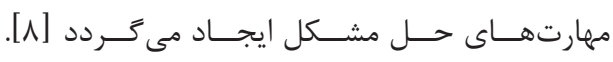

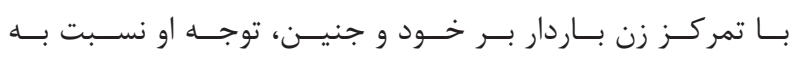

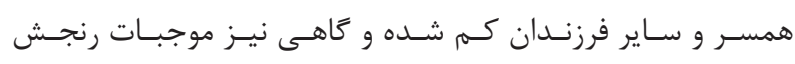

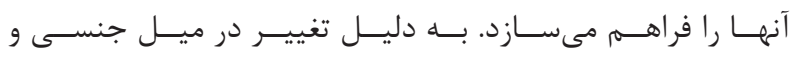

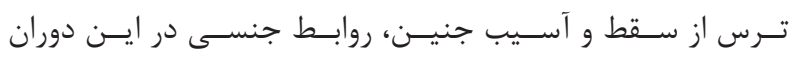

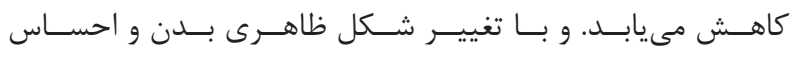

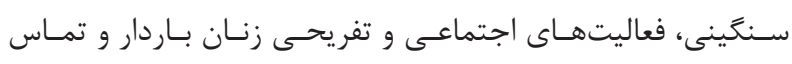

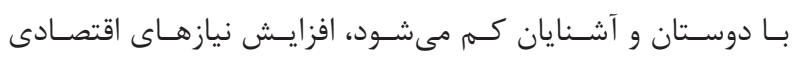

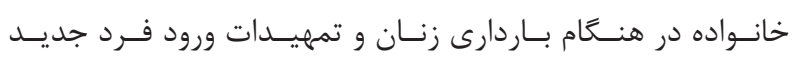

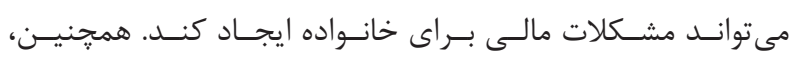

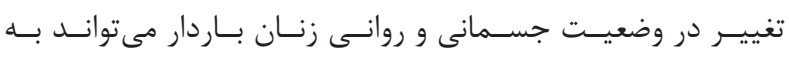

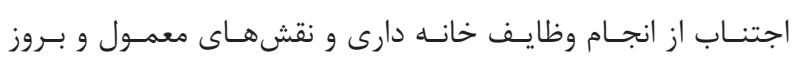

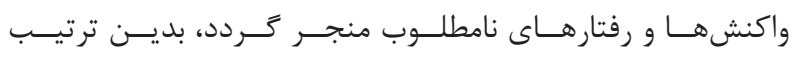

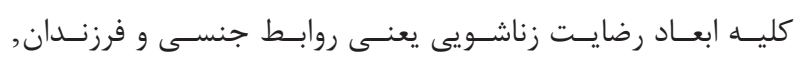

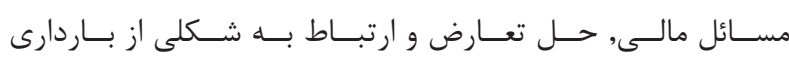

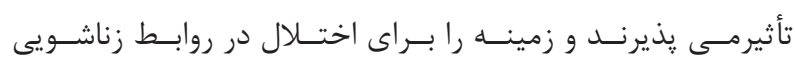

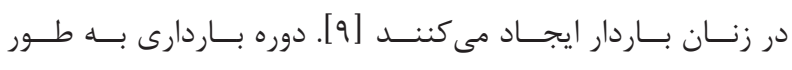

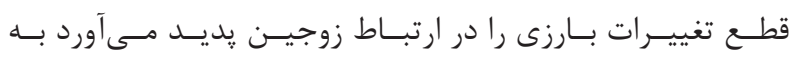

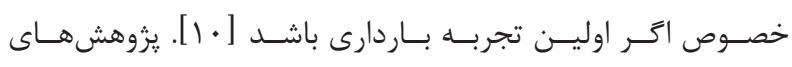

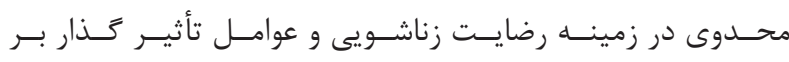

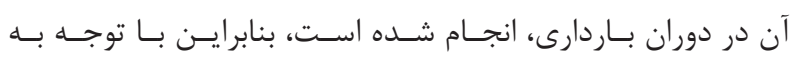

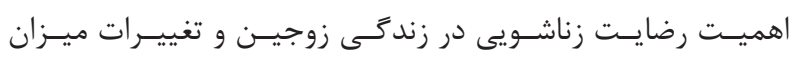

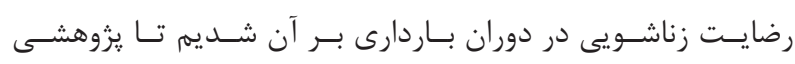

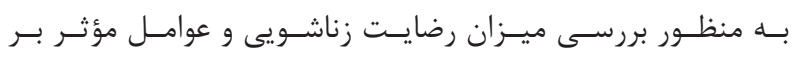

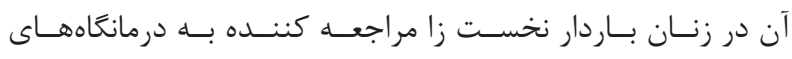

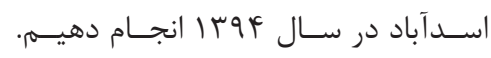




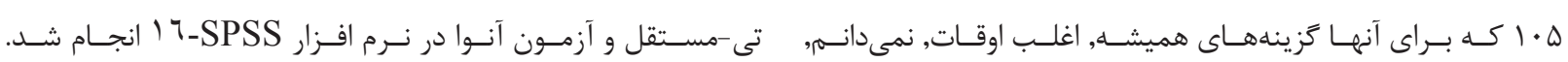

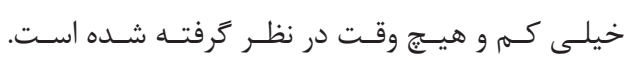

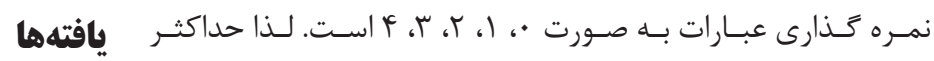

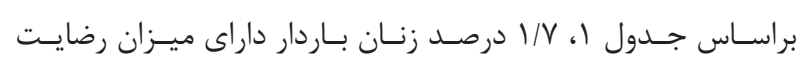

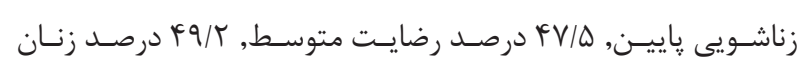

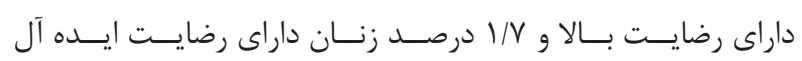

$$
\text { بودنـد. }
$$

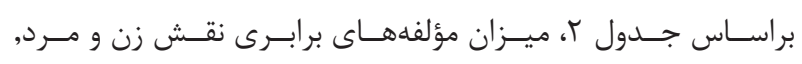

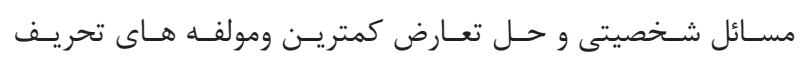

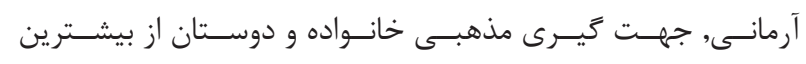

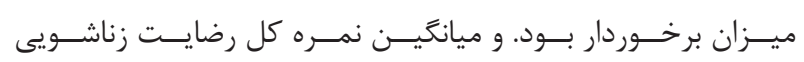

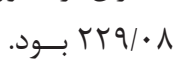

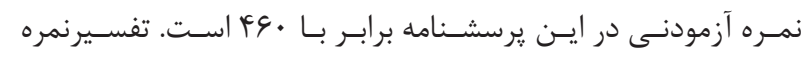

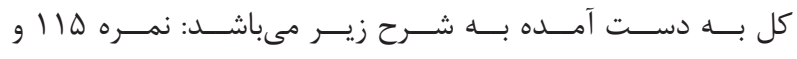

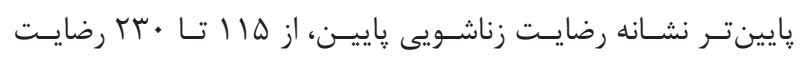

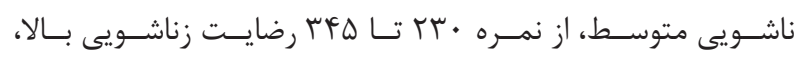

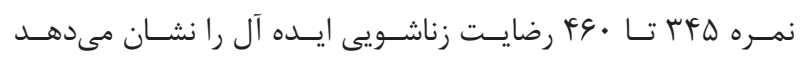

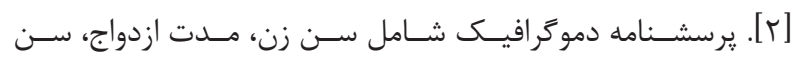

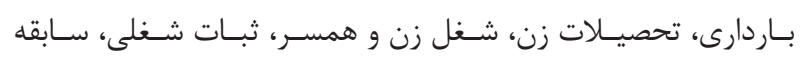

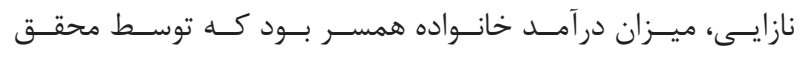

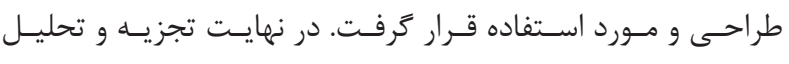

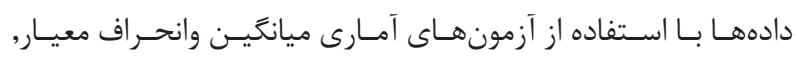

\begin{tabular}{|c|c|c|}
\hline & & جدول ا: توزيع فراوانى سطوح مختلف رضايت زناشويى \\
\hline درصد & تعداد & سطوح رضايت زناشويى \\
\hline $1 / V$ & r & يايين \\
\hline$F V / \Delta$ & $\Delta V$ & متوسط \\
\hline$r q / r$ & $\Delta 9$ & بالا \\
\hline $1 / V$ & r & ايده آل \\
\hline
\end{tabular}

\begin{tabular}{|c|c|}
\hline & جدول r: ميانگين و انحراف معيار متغييرهاى رضايت زناشويى در زنان باردار \\
\hline انحراف معيار 土 ميانگين & متغيير \\
\hline$r F / r \Delta \pm q / 1)$ & تحريف آرمانى \\
\hline$r T / F Y \pm V / I I$ & رضايت زناشويى \\
\hline$|V / \Delta \Delta \pm V / 9|$ & 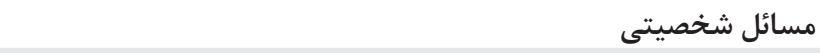 \\
\hline $\mid N / F \Delta \pm V / \varphi$ & ارتباط زناشويى \\
\hline $\mathrm{VV} / \cdot \wedge \pm 9 / \mathrm{VV}$ & حل تعارض \\
\hline$r \cdot / r \mid \pm V / I f$ & مديريت مالى \\
\hline $19 / \Lambda \Lambda \pm \Delta / V F$ & فعاليتهاى اوقات فراغت \\
\hline$r \cdot / r \Delta \pm q / 1 \cdot$ & رابطه جنسى \\
\hline$r Y / \Delta \varphi \pm 9 / 1$. & فرزنديرورى \\
\hline$r r / 9 \cdot \pm 9 / 9 r$ & خانواده و دوستان \\
\hline $11 / r \varepsilon \pm V / \cdot r$ & 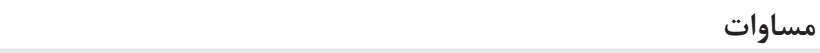 \\
\hline$r r / F V \pm F / \mathcal{F} \Delta$ & جهت گيرى مذهبى \\
\hline$r r q / \cdot \Lambda \pm \Delta r / r \Lambda$ & نمره كل رضايت زناشويى \\
\hline
\end{tabular}




\begin{tabular}{|c|c|c|c|c|}
\hline \multirow[b]{2}{*}{$\mathbf{P}$} & \multirow[b]{2}{*}{ df } & & \multicolumn{2}{|c|}{ جدول ب: ارتباط مشخصههاى دموكرافيك و رضايت زناشويى زنان باردار } \\
\hline & & $\mathbf{t}$ & انحراف معيار ـ ميانَين & سطوح \\
\hline \multirow[t]{5}{*}{.$/ \cdot r$} & r & $\Delta / 1 F$ & & 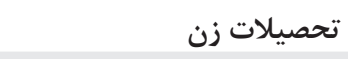 \\
\hline & & & $r T \cdot / r \pm \Delta V / Q V$ & زيردييلم \\
\hline & & & $r|\varepsilon / r \Delta \pm \Delta| / \Delta \Lambda$ & دييلم \\
\hline & & & $r T q / 1 T \pm \Delta 9 / 9 T$ & فوق دييلم \\
\hline & & & $r Q \Delta / 9 T \pm T V / V T$ & 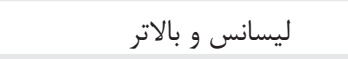 \\
\hline \multirow[t]{3}{*}{$\cdot / \cdot r$} & 111 & $r / \cdot r$ & & 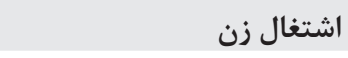 \\
\hline & & & $r V I / r \Lambda \pm G T / F Y$ & شاغل \\
\hline & & & $T Y Y / / D \pm \Delta T / \cdot V$ & 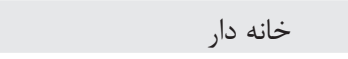 \\
\hline \multirow[t]{4}{*}{$\cdot / \cdot \Delta$} & r & $\Delta / F F$ & & اشتغال همسر \\
\hline & & & $K F N / \Delta D \pm F q / g r$ & 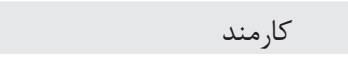 \\
\hline & & & $r r \Delta / V \varphi \pm r q / \wedge \varepsilon$ & شغل آزاد \\
\hline & & & $r \cdot \Delta / \cdot \pm \varphi \Delta / \Lambda 1$ & بيكار \\
\hline \multirow[t]{3}{*}{$\cdot / \cdot 1$} & 111 & $r / \uparrow \varphi$ & & ثبات شغلى همسر \\
\hline & & & $T F \Delta / q T \pm \Delta \cdot / \Lambda \Delta$ & داراى ثبات شغلى \\
\hline & & & TIT/G. $\pm \Delta F / T r$ & بدون ثبات شغلى \\
\hline \multirow[t]{4}{*}{$<\cdot 1 \cdot \cdot 1$} & r & $\mid r / T$ & & ميزان در آمد ماهيانه \\
\hline & & & $r \cdot V / \Lambda \mid \pm \Delta \Lambda / \Delta \Lambda$ & كمتر از • له هزار \\
\hline & & & $r r q / \cdots \pm r \Lambda / q r$ & بين · ·ـ هزار تا 99 هزار \\
\hline & & & $r \varepsilon \Lambda|\cdot \Delta \pm r \varepsilon| \Lambda \mid$ & يك ميليون و بالاتر \\
\hline \multirow[t]{4}{*}{$\cdot / \cdot r$} & r & ए/१९ & & سن (سال) زن \\
\hline & & & $r r \Delta / \Delta \cdot \pm \Delta r / \cdot \Delta$ & IV-rt \\
\hline & & & TrN/IV $\pm \Delta \cdot / 9 \varphi$ & $r r^{-r q}$ \\
\hline & & & $r \cdot r / V F \pm \Delta q / 1 q$ & rq-rq \\
\hline \multirow[t]{3}{*}{$\cdot / \cdot r$} & 111 & $r / \wedge$ & & مدت ازدواج \\
\hline & & & $r r F / q r \pm \Delta r / \cdot r$ & $\cdot-\Delta$ \\
\hline & & & 1८१/१९ $\pm \Delta r / 9 r$ & $\Delta-1 \cdot$ \\
\hline \multirow[t]{3}{*}{$\cdot / \cdot v$} & 111 & r/VT & & باردارى برنامه ريزى شده \\
\hline & & & $r \Psi V / \Lambda \varphi \pm \Delta \cdot / \cdot \Lambda$ & بله \\
\hline & & & $r \cdot N / r \cdot \pm q 1 / r r$ & خير \\
\hline \multirow[t]{3}{*}{$\cdot / \cdot \wedge$} & 111 & $r / 99$ & & سابقه نازايى \\
\hline & & & $r \cdot 1 / F \Delta \pm \varepsilon r / \cdot r$ & بله \\
\hline & & & $r r \Delta / \Delta I \pm \Delta I / \cdot \Lambda$ & خير \\
\hline
\end{tabular}

مطالعـه حاضـر، اكثريـت زنان بـاردار از رضايت زناشـويى متوسـط رو

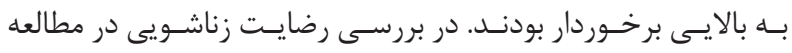

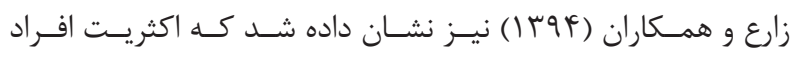

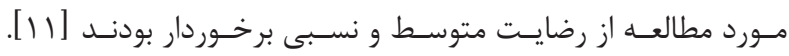

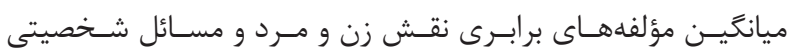

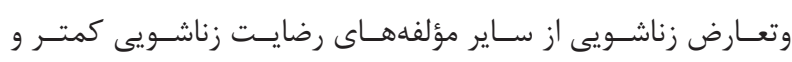

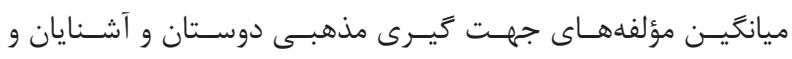

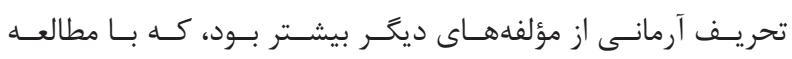

براسـاس جـدول شــماره ب، مشـخصههاى تحصيـلات زن؛, اشـتغال

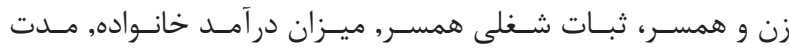

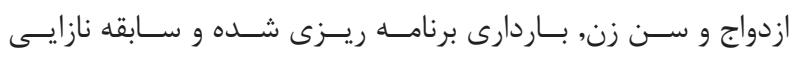

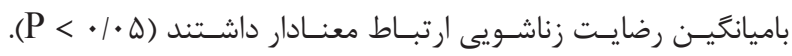
در ايسن يزووهـش بــه بررسـى ميـزان رضايست زناشـويى و تعـدادى

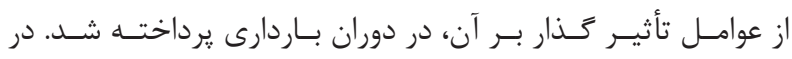




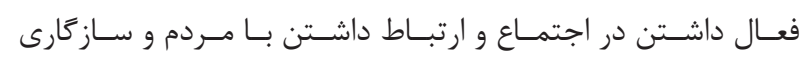

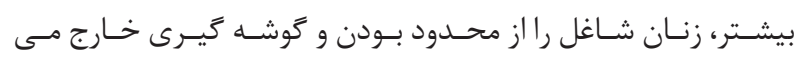

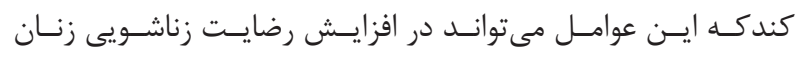

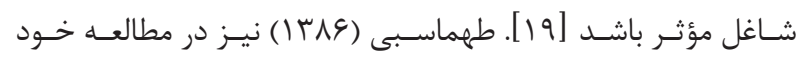

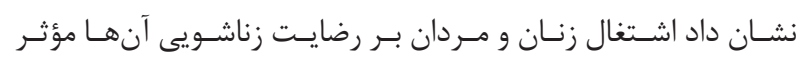

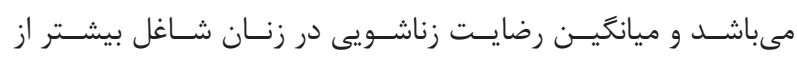

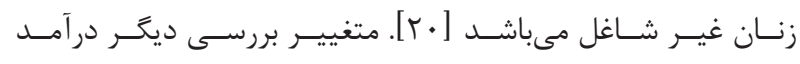

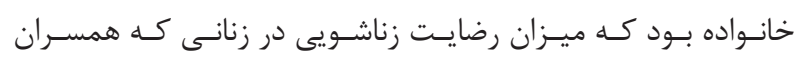

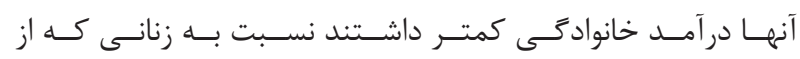

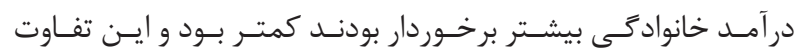

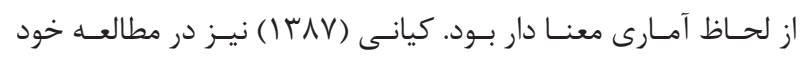

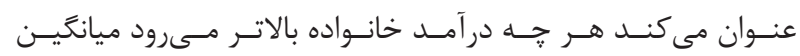

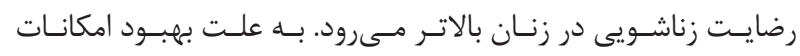

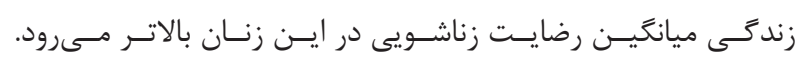

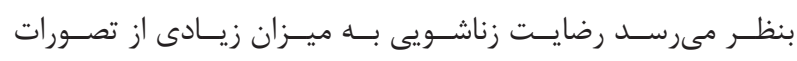

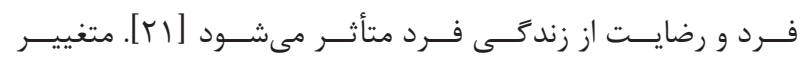

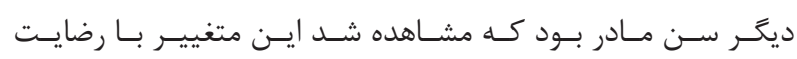

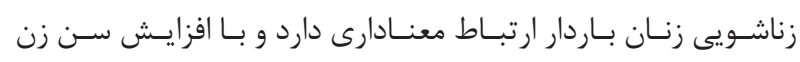

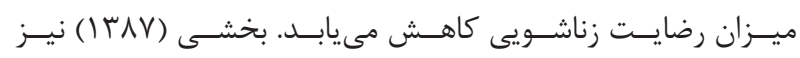

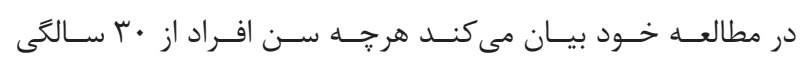

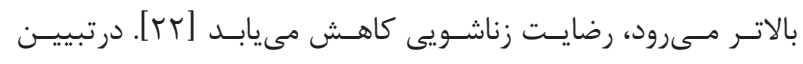

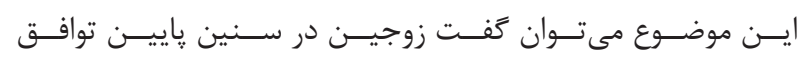

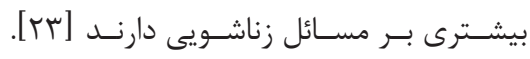

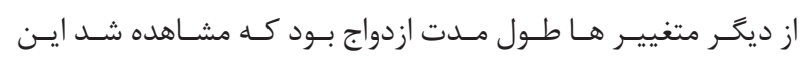

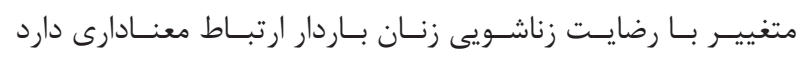

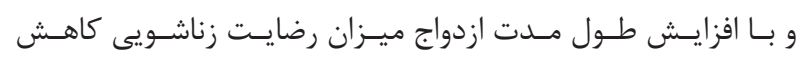

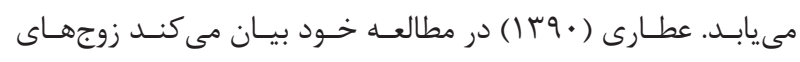

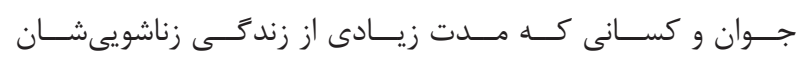

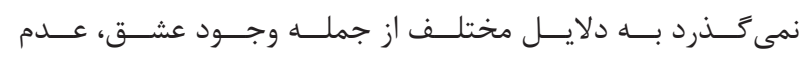

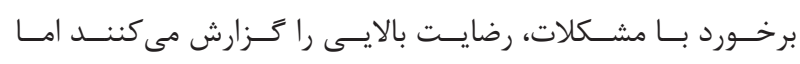

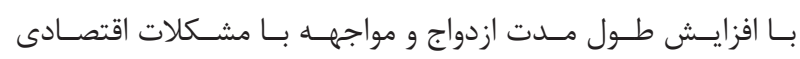

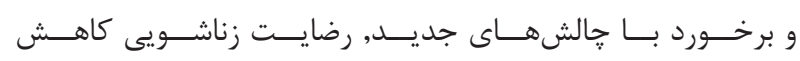

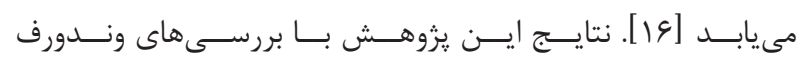

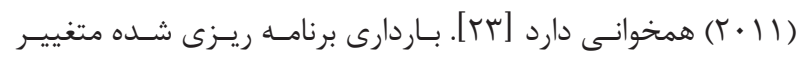

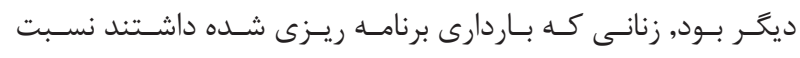

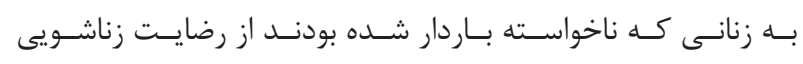

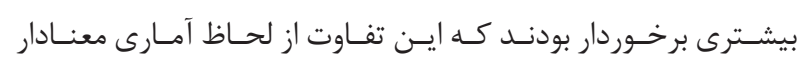

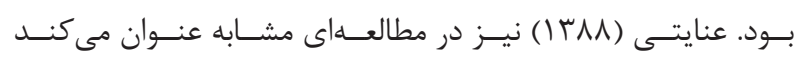

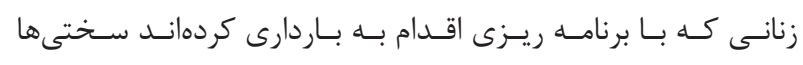

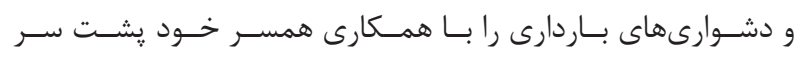

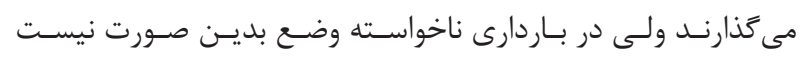

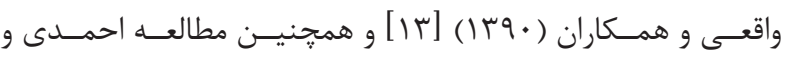

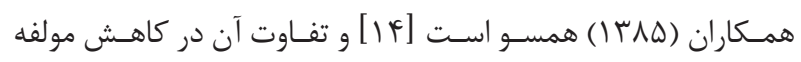

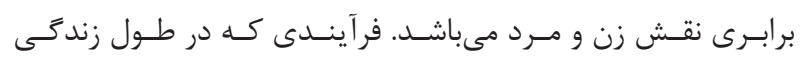

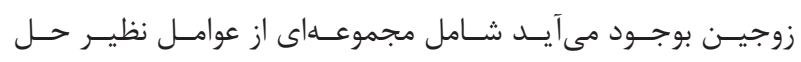

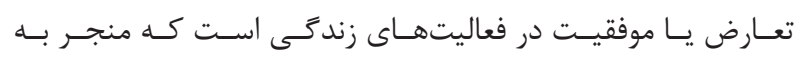

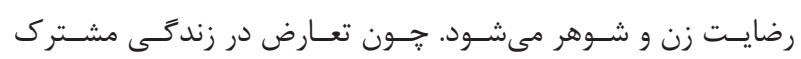

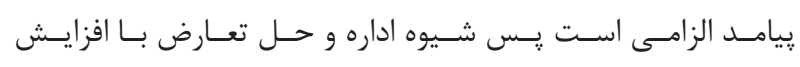

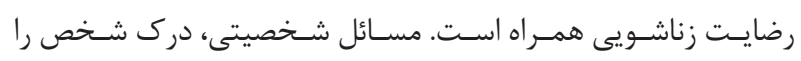

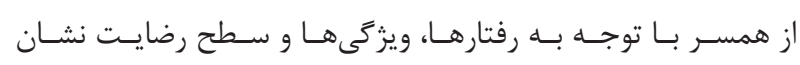

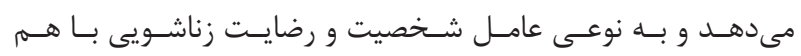

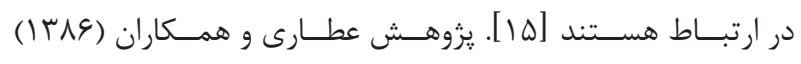

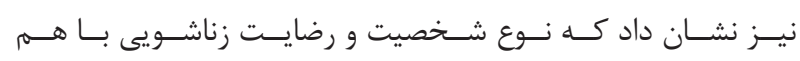

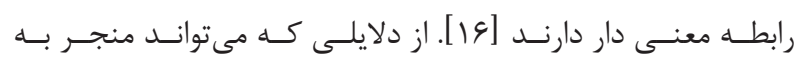

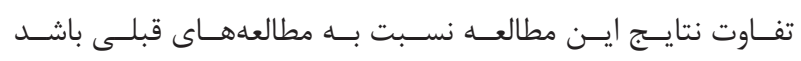

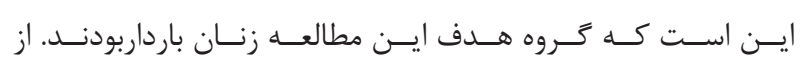

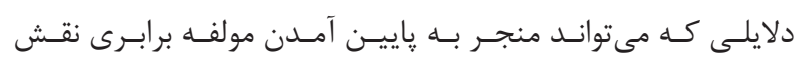

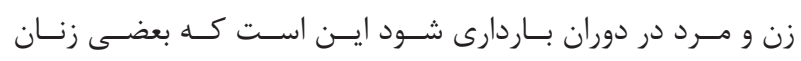

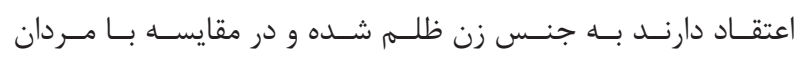

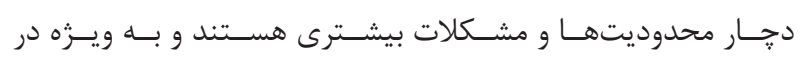

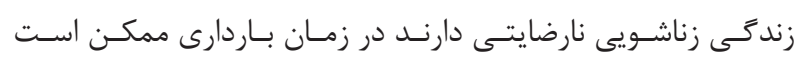

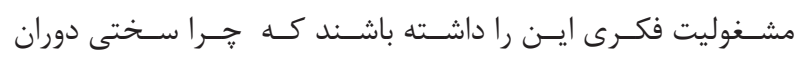

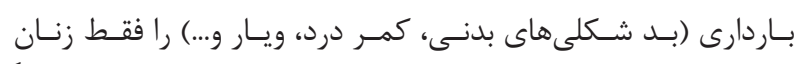

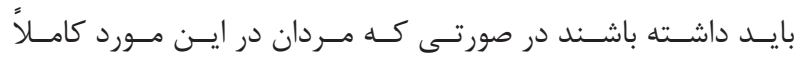

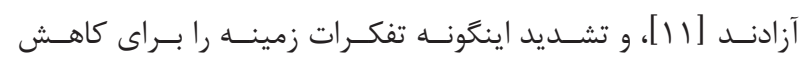

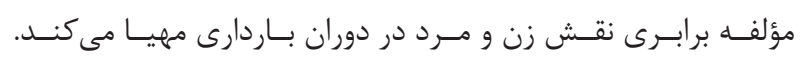

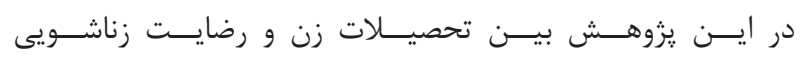

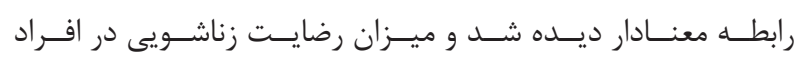

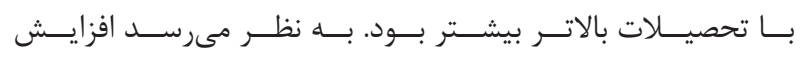

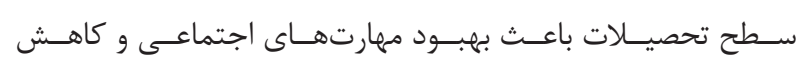

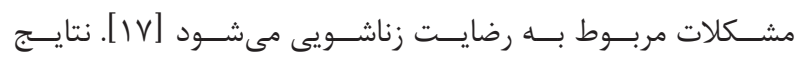

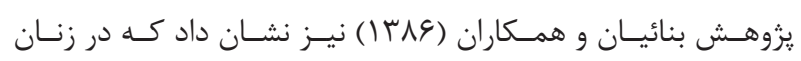

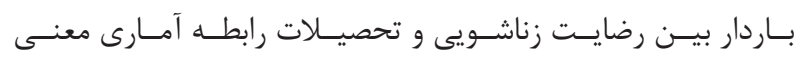

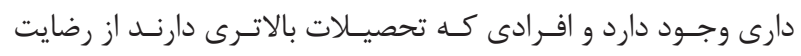

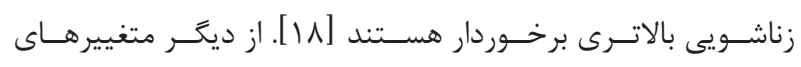

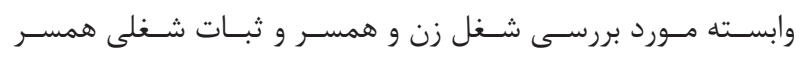

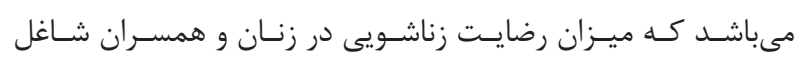

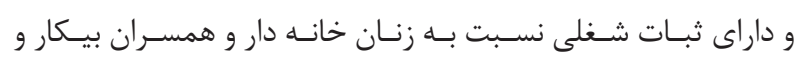

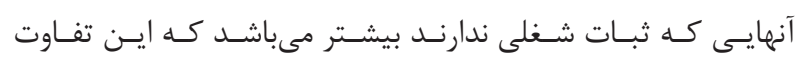

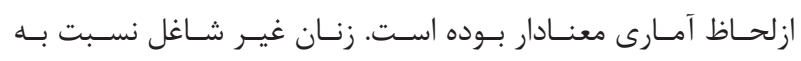

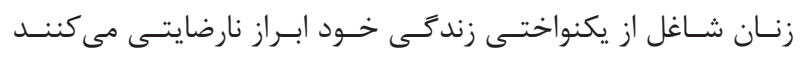

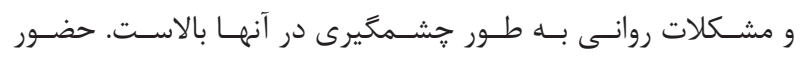




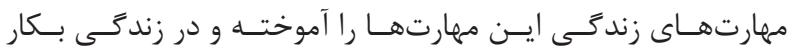

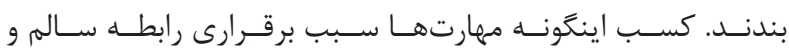

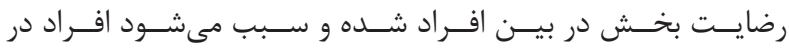

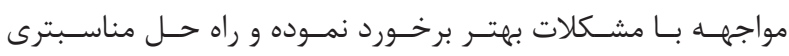

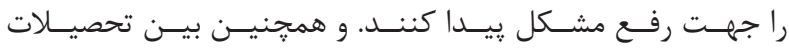

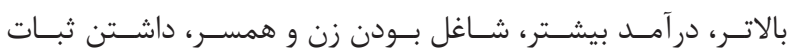

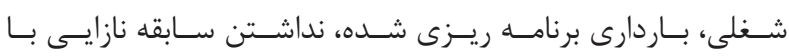

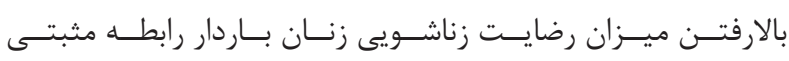

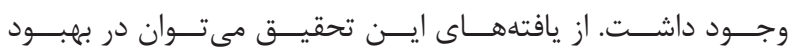

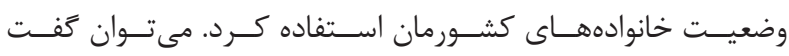

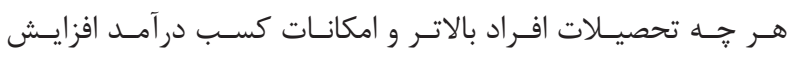

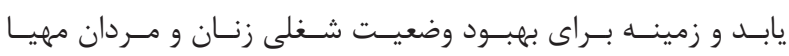

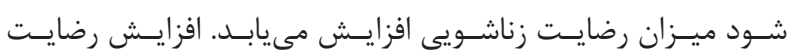

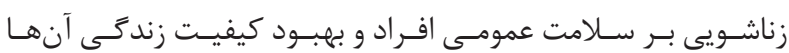

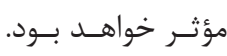

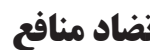

در اين مطالعه تضاد منافع وجود ندارد.

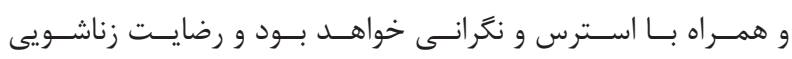

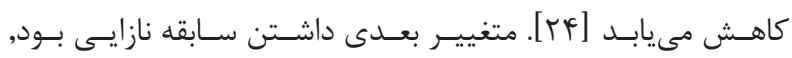

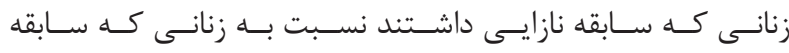

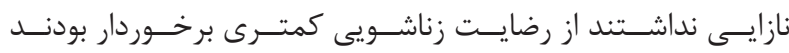

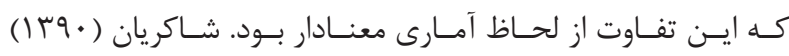

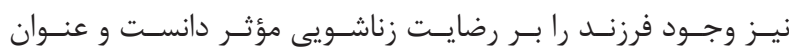

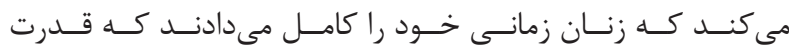

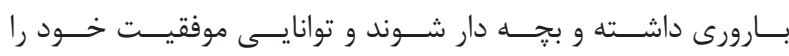

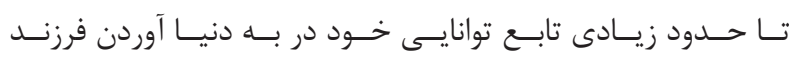

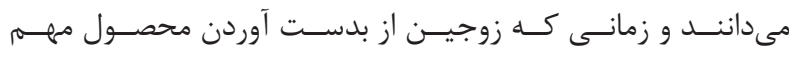

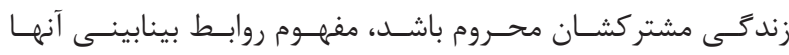

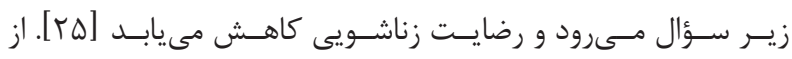

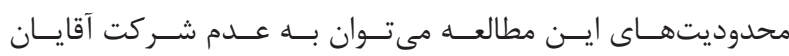

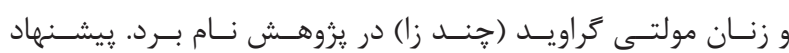

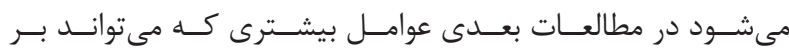

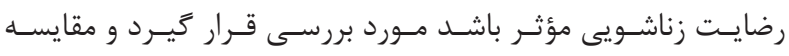

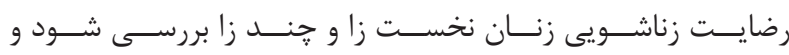

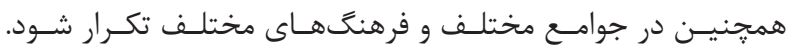

\section{نتيجه گيرى}

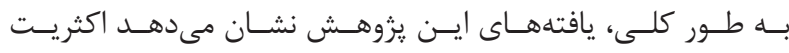

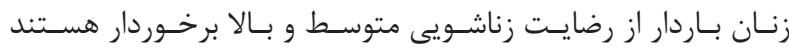

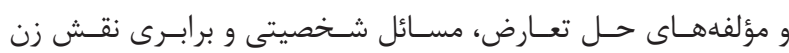

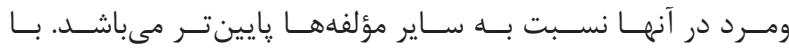

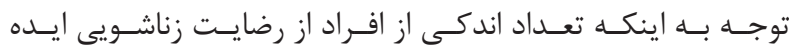

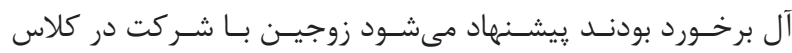

\section{REFERENCES}

1. Jabari Z, Hashemi H. [Effectiveness of cognitive-behavioral stress management techniques on stress anxiety and depression in pregnant women]. J Health Syst Facts. 2011;8(7):1341-7.

2. Khanabadi M, Anbari-Meybodi N, Mahdizadeh F. [Efficacy of the Education Based on the Integration of Transactional-cognitive Analysis on Marital Satisfaction]. Armaghane Danesh. 2015;20(7):63950.

3. Rosand GM, Slinning K, Eberhard-Gran M, Roysamb E, Tambs K. Partner relationship satisfaction and maternal emotional distress in early pregnancy. BMC Public Health. 2011;11:161. DOI: 10.1186/1471-2458-11-161 PMID: 21401914

4. Biehle SN, Mickelson KD. Preparing for parenthood: How feelings of responsibility and efficacy impact expectant parents. J Soc Pers Relat. 2011;28(5):668-83. DOI: 10.1177/0265407510385493

5. Schumacher JA, Leonard KE. Husbands' and wives' marital adjustment, verbal aggression, and physical aggression as longitudinal predictors of physical aggression in early marriage. J Consult Clin Psychol. 2005;73(1):28-37. DOI: 10.1037/0022-006X.73.1.28 PMID: 15709829

6. Kaneto H, Matsuoka T-a, Nakatani Y, Kawamori D, Matsuhisa M, Yamasaki Y. Oxidative Stress and the JNK Pathway in Diabetes. Curr Diabetes Rev. 2005;1(1):65-72.DOI: 10.2174/1573399052952613

7. Pourheydari S, Bagherian F, Doustkam M, Bahadorkhan J. [The Effects of Life Skills Training on Marital and Sexual Satisfaction of

Young Couples]. Knowledge Res Appl Psychol. 2013;14(1):14-22. 8. Delgosha Z. [Maternity and newborn nursing]. 1st ed. Tehran: Sale$\mathrm{mi} ; 2006$.

9. Mangeli M, Ramezani T, Mangeli S. [The effect of educating about common changes in pregnancy period and the way to cope with them on marital satisfaction of pregnant women]. Iranian J Med Educ. 2009;8(2):305-13.

10. Aghayosefi A, Moradi K, Safari N, Ghazi Sh AF. [The study of relationship between marital satisfaction and physical problems during pregnancy and the related factord in pregnant women in Khorramabad city]. J Lorestan Univ Med Sci. 2001;13(1):121-31.

11. Zare B, Safyary Jafar Abad H. [Study of the factors influencing marital satisfaction between men and women in Tehran]. J Women Stud. 2015;13(1):111-40.

12. Fereydoni KJ, Tabrizi M, Nejad SN. [The impact” Self Regulation Brief Couple Therapy" increase couple's satisfaction in the Clients of babolsar Farhangian Clinic]. J Fam Res. 2009;4(15):213-29.

13. Wagheiy Y, Miri M, Ghasemipour M. [A survey about effective factors on the marital satisfaction in employees of two Birjand universities]. J Birjand Univ Med Sci. 2009;16(4):43-50.

14. Ahmadi K, Azad Marzabady E, Mollazamani A. [The study of marital adjustment in Islamic revolutionary guard corps (Sepah) staff]. J Mil Med. 2005;7(2):141-52.

15. Askarian Omran S, Sheikholeslami F, Tabari R. [Effective Factors 
on the Marital Satisfaction in Nurses]. J Guilan Univ Med Sci. 2016;25(97):46-55

16. Attari Y, Amman Elahi A, Mehrabi Zadeh M. [Investigate the relationship between personality characteristics and interpersonal factors Family and marital satisfaction in Ahvaz city]. J Educ Psychol. 2007;3(1):81-108.

17. Jamali S, Zarei H. [Marital Satisfaction in Working and Non-working Pregnant women]. Aligoodarz Nurs Fac Anal Res J. 2014;4(6):57-64

18. Banayeiyan S. [Investigated the relationship between mental health and marital satisfaction married women]. Hamdan J Nurs Midwifery. 2006;14(2):52-62.

19. Motavalli R, Ozgoli G, Bakhtiari M, Alavi Majd H. [Marital satisfaction and marital Intimacy in employed and unemployed pregnant women of Ardebil City]. J Ardabil Univ Med Sci. 2009;9(4):315-24.

20. Tahmasbi S, Ghodsi J, Alavi A, Moradi M. [Comparative study of marital satisfaction for working women and housewives]. J Hamdan Univ Med Sci. 2006;2(14):20-5.
21. Kyani F, Madadzadeh N. [Relationship between marital satisfaction anddemographic variables in postpartum depression(PPD) Astara]. Q J Women Hyg. 2007;1(2):21-32.

22. Bakhshi H, Asadpour M, Khodadadizadeh A. [Correlation between marital satisfaction and depression among couples in Rafsanjan]. J Qazvin Univ Med Sci Health Serv. 2007;2(11):37-43.

23. Craig A, Wendorf Todd Lucas E, Olcay I, Carol C. Satisfaction Across three culture: does the number of children have an impact after Accounting for other marital demographics? J Cross Cult Psychol. 2010;42(3):340-54. DOI: 10.1177/0022022110362637

24. Enayati M, Abd Alrahmi F. [A comparison of mental health and marital satisfaction between'wanted'and'unwanted'pregnancy women referred to the paeental care center in ahvaz]. J Soc Psychol. $2008 ; 2(6): 66-80$.

25. Shakerian A. [Evaluation of the factors influencing marital satisfaction in the students of Islamic Azad Uiversity in Sanandaj]. J Kordestan Univ Med Sci. 2010;14(54):40-9. 


\title{
The Status and Marital Satisfaction Factors in Nul- liparous Pregnant Females Attending Clinics in Asadabad City during Years 2015 and 2016
}

\author{
Batool Khodakarami ${ }^{1}$, Seyyed Zahra Masoomi ${ }^{2,}$, Roya Asadi ${ }^{3}$ \\ ${ }^{1}$ Instructor, Department of Midwifery, School of Nursing and Midwifery, Hamadan \\ University of Medical Sciences, Hamadan, Iran \\ ${ }^{2}$ Assistant Professor, Mother and Child Care Research Center, School of Nursing and \\ Midwifery, Hamadan University of Medical Sciences, Hamadan, Iran \\ ${ }^{3}$ Student, Department of Midwifery, School of Nursing and Midwifery, Hamadan \\ University of Medical Sciences, Hamadan, Iran
}

* Corresponding author: Seyyedeh Zahra Masoomi, Assistant Professor, Mother and Child Care Research Center, School of Nursing and Midwifery, Hamadan University

DOI: $10.21859 / \mathrm{nmj}-25017$ of Medical Sciences, Hamadan,Iran.E-mail: Zahramid2001@yahoo.com

Received: 23.05.2016

Accepted: 16.06 .2016

\section{Keywords:}

Marital Satisfaction

Pregnancy

Personal Information

How to Cite this Article:

Khodakarami B, Masoomi S Z, Asadi R. The Status and Marital Satisfaction Factors in Nulliparous Pregnant Females Attending Clinics in Asadabad City during Years 2015 and 2016. Sci J Hamadan Nurs Midwifery Fac. 2016;25(1):52-59. DOI: $10.21859 / \mathrm{nmj}-25017$

(C) 2017 Scientific Journal of Hamadan Nursing \& Midwifery Faculty

\section{Abstract}

Introduction: Pregnancy is an important event that occurs in the lives of most females. Marital satisfaction is a very important aspect in this period that influences quality of life and physical and mental health of females and their children. Marital satisfaction is influenced by a variety of factors. In this study, we investigated the factors influencing marital satisfaction during this period.

Methods: This descriptive study was conducted on 120 cases that were randomly selected among pregnant females attending clinics of Asadabad during year 2015. Data was gathered by an information form, Enrich Marital Satisfactions Questionnaires and demographic questionnaires. Questionnaires were filled by the participants. The data was analyzed by SPSS 16 software using mean and standard deviation, independent t test, and Analysis of Variance (ANOVA).

Results: Level of marital satisfaction in pregnant females was medium and high. Sub scales of conflict resolution and character issues and equality were lower than the rest of the scales. Subscales of ideal distortion, relatives and friends, and religious orientation were higher than the rest of the scales. Also, there was a significant relationship between education, career, husband's occupation, household income, job stability of the spouse, planned pregnancy, infertility history, length of marriage, age of the woman and marital satisfaction $(\mathrm{P}<0.05)$.

Conclusions: Life skills counseling can be effective in improving marital satisfaction. Demographic variables were predictors of marital satisfaction in pregnant females. 